\title{
Producción de un inoculante a base de rizobacterias promotoras del crecimiento vegetal
}

Blanca Contreras-Roa ${ }^{1} \mid$ Sandra López-Pérez ${ }^{1} \mid$ Juan Pablo Reyes $^{1}$ | Diana Cárdenas-Caro²

Recibido:

Agosto 20 de 2010

Aceptado:

Noviembre 10 de 2011

\section{Resumen}

Los microorganismos promotores del crecimiento vegetal son una alternativa en la biofertilización de los cultivos. Sin embargo, su utilización a nivel comercial requiere del desarrollo de un bioproducto que sea competitivo al nivel de costos y efectos sobre las plantas, con respecto a los fertilizantes tradicionales. Por tal razón, el objetivo de este trabajo fue realizar el estudio de la cinética del crecimiento celular de dos especies bacterianas, Bacillus megaterium BSF5 y Sphingomonas paucimobilis BFS3, coleccionadas en el banco de cepas del laboratorio de Microbiología de la Universidad Francisco de Paula Santander, que se obtuvieron a partir de muestras de suelos de rosa y clavel del invernadero "Flores Iscalá", para producir un inoculante biológico para el mismo cultivo. Se evaluó un medio de cultivo líquido definido y se suministraron diferentes caudales de aire (VVM) con el fin de determinar la velocidad específica de crecimiento $(\mu)$ y la concentración celular en la máxima fase exponencial. A partir de estos resultados se continuó con el diseño de un medio de cultivo alternativo en el cual se suministraron fuentes nutricionales de bajo costo y fácil disponibilidad en la región, como la melaza, úrea y harina de hueso. Se determinó el crecimiento celular en dos concentraciones de azúcares teniendo en cuenta la cantidad que había sido suministrada en el medio de cultivo definido previamente evaluado. Se determinó que la inclusión de melaza en $1{ }^{\circ}$ Brix para Bacillus megaterium y $2^{\circ}$ Brix para Sphingomonas paucimobilis favorece la producción de biomasa y disminuye los costos de materia prima, con respecto, a la obtenida en un medio de cultivo definido.

Palabras clave: Bacillus megaterium, Sphingomonas paucimobilis, inoculantes, bioproceso, biofertilizantes.

\section{Abstract}

The plant growth promoting microorganisms are an alterative in the biofertilization of crops. However, their use a commercial level requires the 


\section{Producción de un inoculante a base de rizobacterias promotoras del crecimiento vegetal}

development of an economic and effective competitive bioproduct compared with the tradicional fertilizers. For this reason, the aim of this work was to perform the kinetics study of two bacterial species Bacillus megaterium BSF5 and Sphingomonas paucimobilis BFS3, collected in the strain bank of the microbiology laboratory of the Universidad Francisco de Paula Santander, obtained from soil samples of rose and carnation from Flores Iscalá greenhouse, to produce a biological inoculant in order to be use in the same crops. A defined liquid culture medium and several air caudal (VVM) were evaluated to determine de specific velocity of growth $(\mu)$ and the cellular concentration in the maximum exponential phase. The obtained results were used to design an alternative culture media using cheap and available nutritional sources in the region such as molasses, urea and bone-meal. The cellular growth was evaluated in two sugar concentrations according with the quantity used in the preliminary evaluated culture media. It was determined that the use of molasses in 1ํㅡㄹ for Bacillus megaterium and $2^{\circ}$ Brix for Sphingomonas paucimobilis favors the biomass production and reduces the raw material costs compared with the result of the define culture medium.

Keywords: Bacillus megaterium, Sphingomonas paucimobilis, inoculants, bioprocess, biofertilizers

\section{Introducción}

U n biofertilizante es un producto biológico que contiene microorganismos benéficos del suelo, que al ser inoculado en el suelo o semilla para un cultivo, favorece los procesos de nutrición, crecimiento y desarrollo de las plantas mediante diversos mecanismos como el incremento de la disponibilidad de nutrientes (solubilizadores de fósforo, fijadores de nitrógeno), mejoramiento de la eficiencia de absorción y transporte de nutrientes (micorrizas) y producción de fitohormonas y vitaminas (promotores del crecimiento vegetal) (Ramírez et al., 2008).

Su utilización comercial no depende sólo de procesos de aislamiento, conservación y evaluación de estos microorganismos que realizan actividades de promoción del crecimiento de las plantas, sino que también se requiere diseñar procesos de producción que permitan obtener la biomasa microbiana necesaria para la utilización de estos microorganismos como inoculantes biofertilizantes en los suelos cultivados con el objetivo de suplir parcial o totalmente la aplicación de fertilizantes sintéticos. Estos inoculantes pueden obtenerse en condiciones artificiales suministrando a las células microbianas nutrientes de alta calidad analítica. Sin embargo, los costos que estos componentes representan, no permiten que el producto obtenido en un escalamiento piloto e industrial, pueda ser comercializado competitivamente frente a algunos fertilizantes químicos que se encuentran disponibles tradicionalmente en el mercado.

De esta forma es necesaria la evaluación de las condiciones de cultivo óptimas de un microorganismo y lo primero que debe tenerse en cuenta es el diseño de medios de cultivo adecuados, basados en los requerimientos 
nutricionales específicos que soporten el crecimiento del microorganismos, la síntesis de sus componentes celulares, así como la producción de metabolitos primarios y secundarios (Sánchez et al., 2003; Borrego et al., 2004).

Actualmente, para cada género incluso especie microbiana, se han formulado algunos medios de cultivos definidos, particularmente para su aislamiento y multiplicación en laboratorio utilizando componentes analíticos y frecuentemente se utilizan en la producción de biomasa. En este sentido, Díaz de Villegas et al. (2002) evaluaron la producción de sideróforos por la cepa PSS de Pseudomonas aeruginosa en un sistema batch convencional utilizando los medios succinato, glucosa, glutámico y King B, con la adición de reactivos analíticos. Por su parte, Rivera-Cruz et al. (2002) utilizaron un medio de cultivo selectivo de carbón combinado modificado $\left(\mathrm{CCM}_{1}\right)$ para bacterias fijadoras de nitrógeno atmosférico tolerantes a BaP (Benzo(a) pireno) con soluciones nutricionales de calidad analítica para la producción de células de Sphingomonas paucimobilis con el objeto de utilizarlo en la biorremediación de suelo contaminados con petróleo.

Sin embargo, recientemente, se han direccionado los esfuerzos buscando reducir los costos de producción de inoculantes con el objetivo de facilitar su comercialización. Plazas (2007), referenció un nuevo medio de cultivo para la producción de la cepa fosfatosolubilizadora UV1 (Enterobacter aglomerans), modificando la adición de componentes analíticos por fuentes nutricionales de origen mineral que suplementarán los requerimientos de este microorganismo, y reportó, incluso un aumento de dos unidades logarítmicas en la producción de biomasa y la reducción del tiempo de producción a 26 horas con respecto al caldo SRS (Sundara Rao y Sinha, 1963). Así mismo, Torres \& Yepes (2007), formularon un nuevo medio de cultivo para la producción de las cepas AC5 y AC6 (Azotobacter chrococcum) reduciendo un 69\% del tiempo en el medio de cultivo convencional. En otras investigaciones desarrolladas, se han utilizado materias primas de origen agroindustrial como la melaza de caña (Goudá et al., 2001; Cárdenas et al., 2002, Kulpreecha et al., 2009), suero de leche (Cori de Mendoza et al., 2006), vinaza (Díaz et al., 2003) y jugo de guayaba agria (Lara, 2008) para el cultivo en fermentación líquida de bacterias y levaduras, encontrando incrementos en la producción de biomasa y sugiriendo reducción de costos de producción por la utilización de estos subproductos como fuentes de energía, minerales y factores de crecimiento celular.

El objetivo de este trabajo fue diseñar un medio de cultivo para la producción a escala de laboratorio de inoculantes microbianos a base de las cepas bacterianas BSF5 (Bacillus megaterium) y BFS3 (Sphingomonas paucimobilis), utilizando materias primas disponibles en la región que suplan los requerimientos nutricionales de estos microorganismos biofertilizantes aislados de suelos cultivados con clavel y rosas, respectivamente.

\section{Materiales y métodos}

Microorganismos estudiados. Se utilizaron las cepas BSF5 (Bacillus megaterium) y BFS3 (Sphingomonas paucimobilis) aisladas en medio SRSM (Sundara Rao y Sinha, 1963) del cultivo de clavel y rosa del invernadero "Flores Iscalá" de la Universidad Francisco de Paula Santander e identificadas bioquímicamente a través de paneles de BBL Crystal (Reyes et al., 2006). Las cepas fueron activadas sobre cajas con agar Nutritivo conformando un banco de trabajo de 10 cajas por cepa.

Curva de crecimiento de los microorganismos suministrando diferentes flujos de aire (VVM). Con el objetivo de conocer la curva de crecimiento de las dos cepas bacterianas, se utilizó el medio de cultivo 
definido propuesto para Bacillus megaterium, cuya composición es $(\mathrm{g} / \mathrm{L})$ : sacarosa 10 ; $\mathrm{K}_{2} \mathrm{HPO}_{4} 2,5 ; \mathrm{KH}_{2} \mathrm{PO}_{4} 2,5 ;\left(\mathrm{NH}_{4}\right)_{2} \mathrm{HPO}_{4} \cdot 7 \mathrm{H}_{2} \mathrm{O}$ 1,$0 ; \mathrm{MgSO}_{4}^{4} \cdot 7 \mathrm{H}_{2} \mathrm{O} 0,2 ; \mathrm{FeSO}_{4} \cdot 7 \mathrm{H}_{2} \mathrm{O} 0,01 ; \mathrm{y}$ $\mathrm{MnSO}_{4} \cdot 7 \mathrm{H}_{2} \mathrm{O}$ 0,007; $\mathrm{pH} \mathrm{7,0}$ (Todar, 2008). Para la cepa de S. paucimobilis, se utilizó el medio de cultivo SRS (Sundara Rao y Sinha, 1963) sin Púrpura de Bromocresol cuya composición es $(\mathrm{g} / \mathrm{L}): \mathrm{NH}_{4}\left(\mathrm{SO}_{4}\right)_{2} 0,5 ; \mathrm{KCl}$ 0,$2 ; \mathrm{MgSO}_{4} \cdot 7 \mathrm{H}_{2} \mathrm{O} 0,3 ; \mathrm{MnSO}_{4} \cdot \mathrm{H}_{2} \mathrm{O} 0,004$; $\mathrm{FeSO}_{4} \cdot 7 \mathrm{H}_{2} \mathrm{O}$ 0,002; $\mathrm{NaCl} 0,2 ;$ Glucosa 10; Extracto de levadura 0,$5 ; \mathrm{Ca}_{3}\left(\mathrm{PO}_{4}\right)_{2} 5 ; \mathrm{pH}$ 7,2. El crecimiento se estudió durante 36 horas, para determinar el número de células/ $\mathrm{mL}$, según la escala de Mc.Farland, leída en un espectrofotómetro $\lambda 1$, a $650 \mathrm{~nm}$, ajustada para cada microorganismo. Se inocularon $500 \mathrm{~mL}$ de medio de cultivo al $2 \%$ de una suspensión bacteriana ajustada según el tubo 1 de la escala de Mc.Farland que equivale a $3 \times 10^{8}$ células $/ \mathrm{mL}$.

Se evaluaron tres medidas de flujo de aire (VVM: Volumen de aire/Volumen de medio/ Minuto) para determinar el crecimiento bacteriano en cada una de ellas. En el T1 se evaluó $1 \mathrm{VVM}$, en el T2 se suministraron 2 VVM y en el T3, 3 VVM. El To se utilizó como testigo en cultivo estático sin suministro de aire. Se realizaron tres repeticiones para cada tratamiento y la concentración celular se midió en cel $/ \mathrm{mL}$ y se transformó a Log. Se realizó un análisis de varianza utilizando el programa estadístico SPSS 10.0 para Windows, con el fin de estimar los parámetros cinéticos de crecimiento, velocidad específica de crecimiento $(\mu)$ y concentración celular en máxima fase exponencial.

Evaluación del medio de cultivo alternativo para la producción de los inoculantes microbianos de Bacillus megaterium y Sphingomonas paucimobilis. Se realizó un diseño experimental para evaluar dos niveles de melaza como una fuente de carbono económica y disponible en la región con el objetivo de reducir costos en las materias primas utilizadas en el medio de cultivo y se comparó el crecimiento bacteriano frente al medio de cultivo con fuentes nutricionales grado reactivo analítico, conformando así tres tratamientos. La melaza no solamente suministra el carbono sino que además, en ella se encuentran disponibles elementos menores, aminoácidos, vitaminas y algunos otros factores de crecimiento que no estarían presentes en un medio de cultivo sintético si no son adicionados como reactivos químicos analíticos (Gouda et al., 2001). Las fuentes de fósforo y nitrógeno también fueron modificadas por cenizas de hueso de bovino ( 5 $\mathrm{g} / \mathrm{L})$ y úrea respectivamente $(0,5 \mathrm{~g} / \mathrm{L})$ según un medio de cultivo alternativo diseñado para la producción de levadura Candida utilis (Cárdenas et al., 2002).

Teniendo en cuenta los resultados de la curva de crecimiento de cada bacteria en el medio de cultivo definido, se diseñaron dos etapas de inóculos en el bioproceso que permitieran obtener paulatinamente volúmenes mayores de biomasa, teniendo en cuenta que suspender grandes cantidades de células tomadas de medios agarizados convencionales resultaría demasiado costoso, además, el crecimiento bacteriano sólo presentará una fase de adaptación, disminuyendo así, el consumo de energía en volúmenes mayores. Este bioproceso se realizó en erlenmeyers contenidos de $100 \mathrm{~mL}$ como preinóculo, los cuales fueron inoculados al $2 \%$, con una concentración celular de $3 \times 10^{8}$ cél $/ \mathrm{mL}$ según el tubo 1 de la escala de Mc. Farland leída en un espectrofotómetro $\lambda 1$, a $650 \mathrm{~nm}$. El tiempo de crecimiento en etapa de preinóculo fue de $18 \mathrm{~h}$ según la fase exponencial presentada por las bacterias en el estudio de su curva de crecimiento en medio de cultivo sintético. Al cabo de este tiempo el preinóculo fue transferido a $900 \mathrm{~mL}$ en un biorreactor de vidrio con capacidad para 3,75 L y fue mantenido con aireación constante durante $18 \mathrm{~h}$, hasta alcanzar el máximo crecimiento exponencial. ISSN 0122-820X 
adaptaron puntos de entrada de aire, salida de gases, toma de muestras e inoculación. El aireador consistió en un tubo plástico en forma de "L" con un dispersor circular con perforaciones hechas cada centímetro y el caudal de aire fue suministrado con una bomba para acuario y regulado con una valvulilla venoclises.

Se realizaron tres repeticiones para cada tratamiento y la concentración celular se midió en cel $/ \mathrm{mL}$ y se transformó a Log para realizar la curva de crecimiento bacteriano. Se realizó un análisis de varianza utilizando el programa estadístico SPSS 10.0 para Windows, con el objetivo de estimar la concentración celular en máxima fase exponencial y seleccionar la mejor concentración de inclusión de melaza como fuente de carbono en la producción de biomasa bacteriana.

\section{Resultados y discusiones}

Efecto del flujo de aire (VVM) en el crecimiento bacteriano. Según las curvas de crecimiento obtenidas para Sphingomonas paucimobilis, se observó que para todos los volúmenes de aire suministrados, la etapa de adaptación alcanzó las $8 \mathrm{~h}$, a partir de las cuales se registraron diferentes comportamientos en la fase exponencial para cada tratamiento evaluado (Figura 1). Así mismo, cuando se realizó el análisis de varianza a la velocidad específica de crecimiento $(\mu)$ durante la fase exponencial, se encontraron diferencias significativas $(\mathrm{P} \leq 0,05)$ en todos los tratamientos, obteniéndose el mayor valor de $0,74 \mathrm{~h}^{-1}$ cuando se suministró 1 VVM. El tratamiento testigo en cultivo estático presentó el menor valor de $0,013 \mathrm{~h}^{-1}$, indicando que para el cultivo de S. paucimobilis es necesario el suministro de aireación en niveles no superiores a $1 \mathrm{VVM}$, teniendo en cuenta que caudales de 2 y 3 VVM registraron un $50 \%$ menos en la velocidad del crecimiento bacteriano con respecto al tratamiento $\mathrm{T} 1$ (Tabla 1).
Figura 1. Curvas de crecimiento de Sphingomonas paucimobilis en medio de cultivo definido con suministro de diferentes caudales de aire (VMM)

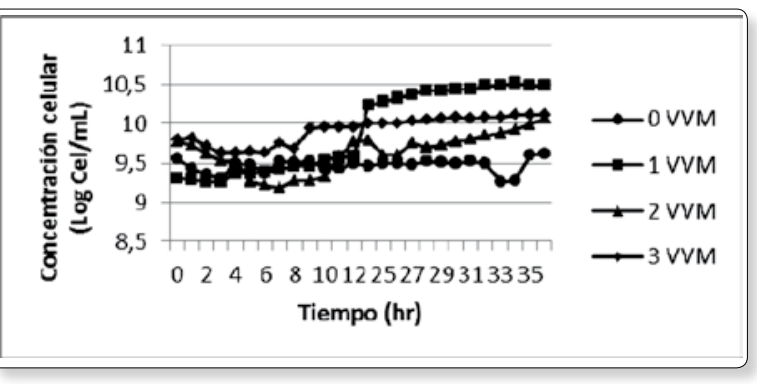

Sánchez et al. (2003), reportaron que Burkholderia cepacia presentó una fase de crecimiento exponencial a partir de las $2 \mathrm{~h}$ de incubación en cultivo agitado a $70 \mathrm{rpm}$ y aunque observaron que a las $8 \mathrm{~h}$ de crecimiento se presentaron los máximos valores de concentración celular, la velocidad específica de crecimiento $(\mu)$ en esta fase exponencial fue de $0,27 \mathrm{~h}^{-1}$, similar a lo registrado en los tratamientos T2 y T3, evaluados para $S$. paucimobilis (Tabla 1 ).

Tabla 1. Velocidad específica de crecimiento $(\mu)$ y concentración máxima celular de Sphingomonas paucimobilis en medio de cultivo definido

\begin{tabular}{|llc} 
Tratamiento & $\boldsymbol{\mu}\left(\mathbf{h}^{-1}\right)$ & $\begin{array}{c}\text { Concentración } \\
\text { celular } \\
(\mathbf{C e ́ l} / \mathbf{m L})^{(1)}\end{array}$ \\
\hline T1 (1VVM) & $0,74 \mathrm{a}^{(2)}$ & $10,34 \mathrm{a}$ \\
T2 (2VVM) & $0,38 \mathrm{~b}$ & $10,03 \mathrm{~b}$ \\
T3 (3VVM) & $0,31 \mathrm{c}$ & $9,79 \mathrm{c}$ \\
T0 (OVVM) & $0,013 \mathrm{~d}$ & $9,57 \mathrm{~d}$ \\
\hline
\end{tabular}

(1) Datos transformados a $\log _{10}$

(2) Valores seguidos con la misma letra en la misma columna no presentaron diferencias significativas por el test de Duncan para un $\alpha=0,05$ de significancia

Giavasis et al. (2006) observaron un incremento en la producción de biomasa de $S$. paucimobilis en un medio de cultivo definido, cuando suministraron 1 VVM y $1000 \mathrm{rpm}$ comparando frente a $1 \mathrm{VVM}$ y $500 \mathrm{rpm}$, pero cuando aumentaron el suministro de aire a 2 VVM y una agitación de 250 a $500 \mathrm{rpm}$ encontraron un incremento en la producción de biomasa, argumentando que el incremento de aireación compensa parcialmente la escasa 
mezcla y transferencia de masa cuando se agita el cultivo a $250 \mathrm{rpm}$ y un poco más en agitación de $500 \mathrm{rpm}$.

Por su parte, Bacillus megaterium presentó un comportamiento muy similar en los cuatro tratamientos evaluados en cuanto a la curva de crecimiento, aunque presentaron diferencias significativas $(\mathrm{P} \leq 0,05)$ y se observó que en el tratamiento con suministro de 1 VVM se obtuvo el mayor valor en la velocidad específica de crecimiento $(\mu)$, con una fase de adaptación de $4 \mathrm{~h}$ y una fase exponencial de máximo 24 h, donde alcanzó una concentración celular de 10,15 Log Cél/ $\mathrm{mL}$ (Figura 2).

Figura 2. Curvas de crecimiento de Bacillus megaterium en medio de cultivo definido con suministro de diferentes caudales de aire (VMM)

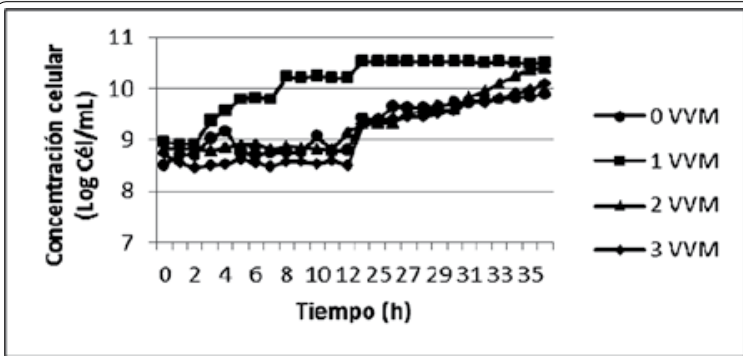

De igual forma que en el cultivo de $S$. paucimobilis, B. megaterium registró un valor de la velocidad específica de crecimiento $(\mu)$ en la fase exponencial de $0,73 \mathrm{~h}^{-1}$, diferente significativamente $(\mathrm{P} \leq 0,05)$ de los demás tratamientos evaluados (Tabla 2), la cual indica que el suministro de 1VVM en el caldo de fermentación, es el más adecuado para el crecimiento celular de estas bacterias con respecto al tiempo de cultivo.

Díaz de Villegas et al. (2002) encontraron valores inferiores de $\mu$ en la fase exponencial en el cultivo de Pseudomonas aeruginosa PA01 en caldos de fermentación utilizando succinato $\left(0,07 \mathrm{~h}^{-1}\right)$, glucosa $\left(0,1394 \mathrm{~h}^{-1}\right) \mathrm{y}$ ácido glutámico $\left(0,064 \mathrm{~h}^{-1}\right)$ en condiciones de agitación de $175 \mathrm{rpm}$, los cuales son similares a los registrados en el tratamiento $\mathrm{TO}$, en cultivo estático para los dos bacterias de este estudio.
Tabla 2. Velocidad específica de crecimiento $(\mu)$ y concentración máxima celular de Bacillus megaterium en medio de cultivo definido

\begin{tabular}{llc} 
Tratamiento & $\boldsymbol{\mu}\left(\mathbf{h}^{-1}\right)$ & $\begin{array}{c}\text { Concentración } \\
\text { celular } \\
\text { (Cél/mL) }^{(1)}\end{array}$ \\
\hline T1 (1VVM) & $0,73 \mathrm{a}^{(2)}$ & $10,15 \mathrm{a}$ \\
T2 (2VVM) & $0,62 \mathrm{~b}$ & $9,36 \mathrm{~b}$ \\
T3 (3VVM) & $0,33 \mathrm{c}$ & $9,32 \mathrm{~b}$ \\
T0 (OVVM) & $0,18 \mathrm{~d}$ & $9,20 \mathrm{~b}$ \\
\hline
\end{tabular}

(1) Datos transformados a $\log _{10}$

(2) Valores seguidos con la misma letra en la misma columna no presentaron diferencias significativas por el test de Duncan para un $\alpha=0,05$ de significancia

Aunque el suministro de aireación en una fermentación líquida pueda aumentar los costos de producción por la energía requerida y el mantenimiento de las condiciones asépticas por el equipo utilizado, debe considerarse que frente al cultivo estático, la producción de biomasa se ve afectada y cuando sólo se suministra agitación, se suple en parte esta dificultad pero no se aumentan significativamente las concentraciones celulares como cuando se suministra aireación. Con respecto a esto, Giavasis et al. (2006) indican que la agitación permite la transferencia de nutrientes y la distribución de aire y oxígeno, pero la aireación determina la oxigenación del cultivo, así como también contribuye a la mezcla de la masa del caldo de fermentación, especialmente cuando las tasas de agitación mecánica son muy bajas. ISSN 0122-820X

Es así como, debe evaluarse la cantidad de aire requerido para el crecimiento celular de una especie bacteriana en determinado medio de cultivo, puesto que no siempre está correlacionado el incremento de la biomasa con el aumento de aireación en una fermentación líquida (Cárdenas et al., 2002). Los resultados obtenidos sugieren que el suministro de aireación es importante para el crecimiento celular de especies bacterianas aeróbicas de igual forma como lo sugiere Giavasis et al. (2006) para el cultivo de S. paucimobilis y Vishnuvardhan et al. (2009) 
para B. megaterium y por lo tanto para la producción de biomasa con fines de desarrollar un inoculante biofertilizante. De esta forma, se seleccionó el tratamiento con suministro de 1 VVM de aireación para el cultivo de las dos bacterias en fermentación líquida.

Selección del medio de cultivo alternativo para la producción de los inoculantes microbianos de Bacillus megaterium y Sphingomonas paucimobilis. La producción de la biomasa bacteriana en caldo de cultivo alternativo presentó mayores valores de Log UFC/mL con respecto a la obtenida en medio de cultivo con reactivos analíticos. $B$. megaterium alcanzó la máxima concentración celular a las $28 \mathrm{~h}$ de proceso, mientras que $\mathrm{S}$. paucimobilis presentó un crecimiento máximo a las 32 horas del proceso (Figura 3).

Figura 3. Crecimiento celular de a) Sphingomonas paucimobilis y b) Bacillus megaterium en medio de cultivo melaza
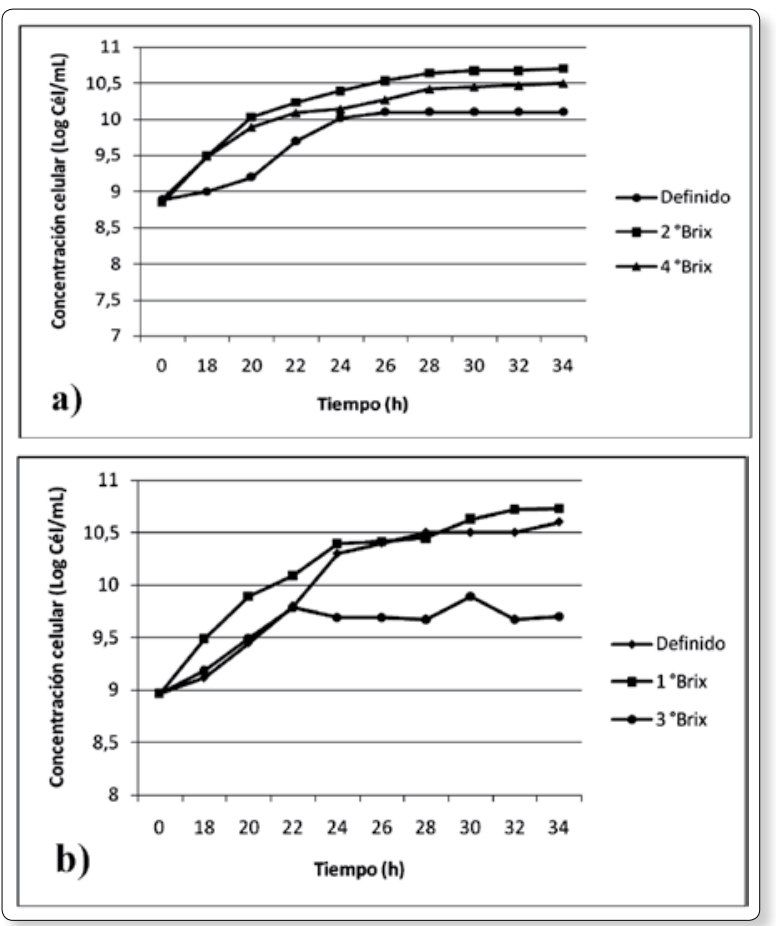

Estos resultados son similares a los obtenidos con otros microorganismos cuando se han adicionado fuentes de carbono complejas como la melaza, extracto de malta, jarabe de maíz, suero de leche y almidón soluble, en comparación con azúcares puros como la glucosa, fructosa y sacarosa ya que los primeros son fuente también de vitaminas, oligoelementos y otros factores requeridos en el crecimiento bacteriano (Nikel et al., 2005; Cori de Mendoza et al., 2006; Kulpreecha et al., 2009).

Cuando se realizó el análisis de varianza y pruebas de comparación múltiple por el test de Duncan, se encontraron diferencias significativas $(\mathrm{P} \leq 0,05)$ en la concentración celular máxima de $\mathrm{S}$. paucimobilis en medio de cultivo con melaza en una concentración de $2^{\circ}$ Brix, con respecto a la concentración de $4^{\circ}$ Brix, lo cual favorece los costos de producción por la disminución de la cantidad de materia prima requerida en el bioproceso (Tabla 3).

Estosresultadossonsimilaresalosreportados por Bajaj et al. (2006) cuando evaluaron diferentes fuentes de carbono como la fructosa, sacarosa, maltosa, lactosa y almidón soluble en una concentración de $2 \%$ y observaron que la sacarosa favoreció la producción de biomasa de $\mathrm{S}$. paucimobilis con respecto a la glucosa y demás fuentes de carbono simples. Sin embargo, cuando utilizaron almidón soluble como fuente alternativa y compleja de carbono, encontraron el mayor crecimiento celular significativamente diferente a todos los cultivos donde se adicionaron las demás fuentes de carbono.

Por su parte, B. megaterium no presentó diferencias $(\mathrm{P} \leq 0,05)$ al comparar la concentración celular máxima en medio de cultivo con reactivos analíticos y medio de cultivo con melaza en la concentración de 1․Brix. Gouda et al. (2001) evaluaron la inclusión de melaza en el caldo de fermentación para la producción de polihiroxibutiratos (PHB) por B. megaterium y registraron que el $3 \%\left(3^{\circ}\right.$ Brix $)$ de inclusión de melaza favoreció el crecimiento bacteriano y cuando se adicionó solo el 2\% de melaza, aumentó la producción de los (PHB). Kulpreecha et al. (2009), encontraron que cuando se adicionó melaza al $2 \%$ ( $2^{\circ}$ Brix) y úrea al 0,8\% se obtuvo mayor producción de biomasa que al cultivar B. megaterium en sacarosa al $2 \%$. 


\section{Producción de un inoculante a base de rizobacterias promotoras del crecimiento vegetal}

Tabla 3. Concentración máxima celular de Bacillus megaterium y Sphingomonas paucimobilis en medio de cultivo definido y alternativo

\begin{tabular}{|c|c|c|c|}
\hline Tratamiento & $\begin{array}{l}\text { Concentración celular de } \\
\text { B. megaterium }(\mathrm{Cél} / \mathrm{mL})^{(1)} \\
(\mathrm{Cél} / \mathrm{mL})^{(1)}\end{array}$ & Tratamiento & $\begin{array}{l}\text { Concentración celular de } \\
\text { S. paucimobilis } \\
(\text { Cél } / \mathrm{mL})^{(1)}\end{array}$ \\
\hline T1 (1 ${ }^{\circ}$ Brix $)$ & $10,72 a^{(2)}$ & $\mathrm{T} 1 \quad\left(2^{\circ}\right.$ Brix $)$ & $10,74 \mathrm{a}^{(2)}$ \\
\hline T2 (3º Brix) & $9,94 b$ & $\mathrm{~T} 2$ (4 ${ }^{\circ}$ Brix $)$ & $10,42 b$ \\
\hline T3 (Definido) & $10,50 \mathrm{ab}$ & T3 (Definido) & $10,20 c$ \\
\hline
\end{tabular}

(1) Datos transformados a $\log _{10}$

(2) Valores seguidos con la misma letra en la misma columna no presentaron diferencias significativas por el test de Duncan para un $\alpha=0,05$ de significancia

La utilización de melaza como fuente de carbono compleja en los medios de cultivo para la producción de S. paucimobilis y B. megaterium incrementó el crecimiento bacteriano con respecto al suministro de glucosa y sacarosa respectivamente los caldos de fermentación con fuentes nutricionales definidas. Esto indica que el crecimiento bacteriano se ve favorecido en el medio alternativo formulado con melaza de caña y puede ser utilizado como un caldo de fermentación a nivel de laboratorio y proyectarse hacia una producción de mayor escala.

Esto ha sido atribuido a que la melaza de caña contiene mayores requerimientos nutricionales como carbohidratos (sacarosa y glucosa) como fuente de energía, componentes nitrogenados (aminoácidos, amonio) para la síntesis de biomasa microbiana, minerales o elementos traza (sodio, azufre, fósforo, calcio, magnesio, potasio, hierro) indispensables para la activación de enzimas, respiración celular y vitaminas (tiamina, riboflavina, piridoxina y niacinamida) como fuente de factores de crecimiento celular (Gouda et al., 2001; Cárdenas et al., 2002; Lara, 2008). La utilización de estos subproductos agroindustriales como principales componentes de los caldos de cultivo, reducen los costos de producción asociados con la elección del sustrato en las fermentaciones industriales, especialmente en países donde la agroindustria tiene un profundo impacto sobre los productos de la canasta familiar (Nikel et al., 2005) y se considera una alternativa viable de sustitución de los medios de cultivo definidos, en los cuales se aumentan los costos de producción y se obtiene menor contenido de biomasa celular necesaria para desarrollar el producto inoculante.

\section{Conclusiones}

El crecimiento celular bacteriano presentó mayores valores cuando se suministró un caudal de aireación de 1 VVM y se precisó un máximo tiempo de proceso de $28 \mathrm{~h}$ para Bacillus megaterium y de $32 \mathrm{~h}$ para Sphingomonas paucimobilis.

Se determinó que el medio de cultivo con inclusión de melaza favoreció la producción de biomasa de las bacterias Sphingomonas paucimobilis y Bacillus megaterium con respecto al medio definido que contiene reactivos grado analítico, lo cual permitirá desarrollar un inoculante a escala de laboratorio con bajo costo de producción. ISSN 0122-820X
Respuestas 


\section{Referencias bibliográficas}

Bajaj, I.B., Saudagar, P.S., Singhal, R.S. and Pandey, A. 2006. Statiscal approach to optimization of fermentative production of gellan gum from Sphingomonas paucimobilis ATCC 31461. Journal of Bioscience and Bioengineering 102(3): 150-156.

Borrego, S., Espinosa, M.E., Martí, E. y Fonseca, M. 2004. Optimización de un medio de cultivo salino para la producción de androstendiona a partir de cholesterol usando la cepa Mycobacterium sp. MB-3683. Biotecnología Aplicada 1: 21-24.

Cárdenas, D.M., Parada, C.M., Muñoz, Y.A. y Moreno, L.Y. 2002. Producción de proteína para alimentación animal mediante bioconversion de sustratos agropecuarios. Revista Respuestas, 7(1): 9-13.

Cori de Mendoza, M.E., Rivas, N., Dorta, B., Pacheco de Delahaye, E. y Bertsch, A. 2006. Obtención y caracterización de dos concentrados proteicos a partir de biomasa de Kluyveromyces marxianus var. marxianus cultivada en suero lácteo desproteinizado. Revista científica, FCV-Luz 16(3): 315-324.

Díaz de Villegas, M.E., Villa, P. and Frías, A. 2002. Evaluation of the siderophores production by Pseudomonas aeruginosa PSS. Rev. Latinoam. Microbiol. 44(3-4): 112-117.

Díaz, M., Semprún, A. y Gualtieri, M. 2003. Producción de protein unicelular a partir de desechos de vinaza. Revista de la Facultad de Farmacia, 45(2): 23-26.

Giavasis, I., Harvery, L.M. and McNeil, B. 2006. The effect of agitation and aeration an the synthesis and molecular weighe of gellan in batch cultures of Sphingomonas paucimobilis. Enzyme and Microbial Technology, 38: 101108.
Gouda, M.K., Swellam, A.E. and Omar, S.H. 2001. Production of PHB by a Bacillus megaterium strain using sugarcane molasses and corn steep liquor as sole carbon and nitrogen sources. Microbiol. Res. 156: 201 207.

Kulprecha, S., Booruangthavorn, A., Meksiriporn, B. and Thongchul, N. 2009. Inexpesive fed-batch cultivation for high poly (3-hidroxybutyrate) production by a new isolate of Bacillus megaterium. Journal of Bioscience and Bioengineering, 107(3): 240245.

Lara, C. 2008. Composición química de un medio de cultivo a partir de guayaba agria (Psidium araca) y su relación con la nutrición de los microorganismos ruminales. Rev. Colomb. Biotecnol. 10(2): 44-49.

Nikel, P.I., Pettinari, M.J., Méndez, B. S. and Galvagno, M.A. 2005. Statiscal optimización of a culture medium for biomass and poly (3-hydroxybutyrate) production by a recombinant Escherichia coli strain using agroindustrial by products. International Microbiology, 8: 243-250.

Plazas, C. 2007. Mejoramiento de un medio de cultivo para la producción de un inoculante con base en bacterias fosfatosolubilizadoras. Tesis de Grado. Microbiología Industrial. Pontificia Universidad Javeriana.

Ramírez, M., Roveda, G., Bonilla, R., Cabra, L., Peñaranda, A., López, M., Tamayo, A., Navas, G.E. y Díaz, C.A. 2008. Uso de microorganismos con potencial como biofertilizantes en el cultivo de mora. Ed. Produmuedios: Bogotá, D.C. 64 pp.

Reyes, J.P., Contreras, B.L., López, S.M. 2006. Obtención de microorganismos nativos con potencial biofertilizante para su aplicación en los cultivos del invernadero "Flores Iscalá" de la Universidad Francisco de Paula Santander. Tesis de Grado. Ingeniería de Producción 
Biotecnológica. Universidad Francisco de Paula Santander. 338 pp.

Rivera-Cruz, M.C., Ferrera-Cerrato, R., Volke-Haller, V., Fernández-Linares, L. y Rodríguez-Vásquez, L. 2002. Adaptación y selección microbiana autóctona en medios de cultivo enriquecidos con Benzo(a)Pireno. Agrociencia, 36(5): 503-514.

Sánchez, L., Ortiz, S. y Hernández, A. 2003. Obtención de sideróforos a partir de Burkholderia cepacia y optimización del medio de cultivo para su producción. Rev. Salud Anim. 25(1): 27-33.

Sundara, R. and Sinha. 1963. Phosphate disolving microorganisms in the soil and rhizosfere. Indian J. Agric. Sci. 33: 272-278.

Todar, K.2008. Nutrition and growth of bacteria. University of Wisconsin- Madison Department of Bacteriology. En: < http:// textbookofbacteriology.net/nutgro_3.html $>$

Torres, T. y Yepes, M. 2007. Evaluación de medios de cultivo líquidos y dos sistemas de separación físicos para dos bacterias del género Azotobacter sp. a nivel de laboratorio. Tesis de Grado. Ingeniería Química. Fundación Universidad de América.

Vishnuvardhan, S. and Thirumala, M. 2009. Production of PHB and P (3HB-co-3HV) biopolymers by Bacillus megaterium strain OU303A isolated from municipal sewage sludge. World J. Microbiol. Biotechnol. 25: 391-397. 